\title{
SURFACE-MICROMACHINED IN-CHANNEL PARYLENE DUAL VALVES FOR UNPOWERED MICROFLOW REGULATION
}

\author{
Po-Jui Chen ${ }^{1}$, Damien C. Rodger ${ }^{1,2}$, Ellis Meng ${ }^{3}$, Mark S. Humayun ${ }^{2}$, and Yu-Chong Tai ${ }^{1}$ \\ ${ }^{1}$ California Institute of Technology, Pasadena, CA 91125 USA \\ ${ }^{2}$ Keck School of Medicine, University of Southern California, Los Angeles, CA 90033 USA \\ ${ }^{3}$ University of Southern California, Los Angeles, CA 90089 USA
}

\begin{abstract}
This paper presents the world's first in-channel parylene dualvalved microfluidic system for unpowered microflow regulation. Incorporating a normally-closed and a normally-open passive check valve in a back-to-back configuration inside a microchannel, the dual-valved system has successfully regulated the pressure/flow rate of air or liquid without power consumption or electronic/magnetic/thermal transduction. By exclusively using parylene $\mathrm{C}$ as the structural material, the fabricated valves have higher flexibility to shunt flows in comparison with other conventional thin-film valves. In addition, the parylene-based devices are completely biocompatible/implantable, and provide an economical paradigm for fluidic control in integrated lab-on-a-chip systems. Testing results have successfully demonstrated that the microflow regulation of the dual-valved system can achieve $\mathrm{mmHg}$ pressure $-\mathrm{mL} / \mathrm{min}$ flow rates for air and $\mathrm{mmHg}$ pressure $\mu \mathrm{L} / \mathrm{min}$ flow rates for water. This regulation range is suitable for controlling biological conditions in human healthcare, such as regulating elevated intraocular pressure (IOP) in glaucoma patients.
\end{abstract}

\section{INTRODUCTION}

Micro-electro-mechanical systems (MEMS) is an enabling technology to build various microfluidic devices (e.g., channels, valves, pumps, mixers, etc.) for micro-total-analysis systems ( $\mu$ TAS) and lab-on-a-chip applications [1][2]. Among such devices microvalves are the key component to systematically control microflow for reliable operation. Microvalves are designed to be active or passive, with passive valves (check valves) having advantages of simple operation, zero power consumption, and ease of integration. Based on their actuation mechanism, micromachined check valves are categorized as normally-open (Fig. 1(a), fluidic channel is always open unless there is sufficient backward pressure) [3] or normally-closed (Fig. 1(b), fluidic channel is blocked until pressure is above cracking pressure) [4][5] valves. However, both types of check valves are not ideal. For normally-open valves the cracking pressure to conduct forward flow is minimal, while there is always a leakage problem when backflow is applied. For normally-closed valves the backflow leakage can be reduced, but the cracking pressure turns out to be so large that reasonable flow rate is not achievable with low pressure loading. These problems are inevitable using current MEMS fabrication technologies.

Even though micromachined check valves are not ideal, different design schemes can be implemented to realize different fluidic behaviors from those in a single valve. In this work, a novel dual-valve configuration has been developed to achieve passive microflow regulation by incorporating a normally-closed check valve and a normally-open check valve together inside a microchannel. This configuration inherently regulates pressure and flow rate of fluids without any power consumption. In addition to implementing similar design concepts from previous

work [3][4], both the design and fabrication of normally-closed and normally-open check valves have been modified and improved so that the in-channel dual-valved system is capable of controlling flows in the $\mathrm{mmHg}$ pressure $-\mu \mathrm{L} / \mathrm{min}$ flow rate range, further controlling biological conditions in human healthcare. For example, a dual-valved device can be implanted in a glaucoma patient's eye to facilitate regulation of his or her elevated IOP. Such a configuration can also be integrated in lab-on-a-chip systems for refined flow regulation in life science research.

\section{DESIGN}

In order to realize flow regulation, the back-to-back dualvalved configuration illustrated in Fig. 1(c) was developed. By placing a normally-closed valve in forward flow operation at the inlet and placing a normally-open valve in backward flow operation at the outlet of a microchannel, the overall fluidic behavior combines the dashed regions of Fig. 1(a) and Fig. 1(b), resulting in the curve shown in Fig. 1(c). In this configuration, the normally-closed check valve dominates the fluidic control in terms of conducting flows through the channel at pressures higher than the threshold point. The normally-open check valve works in reverse leakage mode so that it serves as a fluid stopper, in which flow at pressures higher than the cut-off point is blocked. If the individual check valves are well-tuned, this passive in-channel dual-valved microsystem is able to spontaneously regulate pressure/flow rate in the designated range of forward flows without active actuation. Additionally, this configuration is leakproof to backward flows due to the normally-closed valve at channel inlet. Because the dual valves are required to be integrated in-channel for this back-to-back operation, they were designed to be surface-micromachined for fabrication convenience.

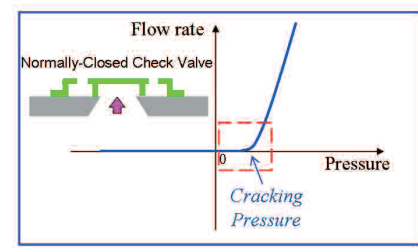

(a)

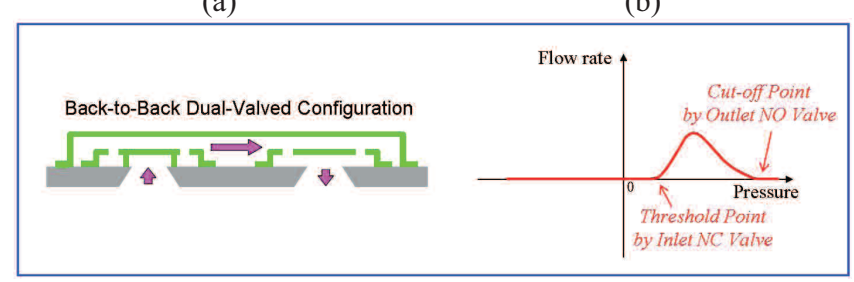

(c)

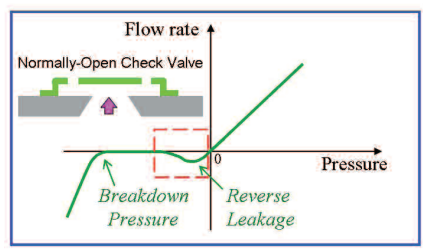

(b)

Figure 1. Concept of dual-valved microflow regulation. Combining the single valve behavior shown in the dashed regions of (a) and (b) in a "back-to-back" configuration results in the passive pressurelflow rate regulation shown in (c). 
Fig. 2 shows the designs of micro check valves. A freestanding membrane suspended by tethers acts as the normallyopen check valve, in which forward flow can be easily achieved while reserve leakage happens in backflow [3]. As for the normally-closed valve, it needs a pre-stressing force to block the inlet orifice so forward flow can not pass through without sufficient pressure loading. Accordingly, a vacuum-collapsed sealing method [4] is implemented. As a chamber is collapsed by a pressure difference between the chamber pressure and the ambient pressure, the diaphragm connected to the chamber is snapped downward and pressed against the valve seat, resulting in the dynamic behavior of a normally-closed check valve. To ensure that the cracking pressure of the normally-closed valve is well controlled, its mechanical design was verified by iterative FEMLAB $^{\mathrm{TM}}$ (now called COMSOL Multiphysics ${ }^{\mathrm{TM}}$ ) simulations (Fig. 3) to estimate the valve performance. Depending on the use of straight-armed or twisted-armed tethers to suspend the membrane, single check valves can have different structural rigidities and their fluidic behaviors (flow resistance, cracking pressure, etc.) are correspondingly altered. Parylene C (poly-paraxylylene $\mathrm{C}$ ) was selected as the structural material because of its mechanical flexibility (Young's modulus $\sim 4 \mathrm{GPa}$ ), chemical inertness, biocompatibility (USP Class VI grade), and CMOS/MEMS process compatibility. Furthermore, conformal parylene deposition takes place at room temperature in a vacuum ( $\sim 22$ mTorr), which facilitates the vacuum-collapsed sealing of the normally-closed check valve.

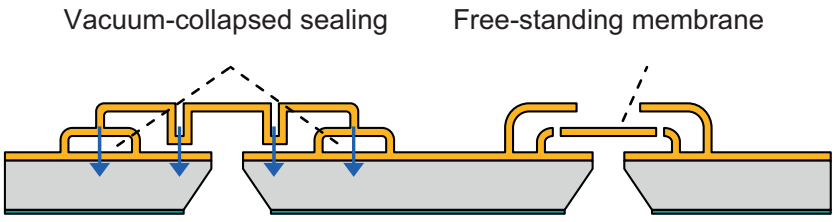

Figure 2. Cross-section of normally-closed (left) and normallyopen (right) micro check valves. Arrows in normally-closed valve denote the dragging direction when the chamber is collapsed by vacuum sealing.

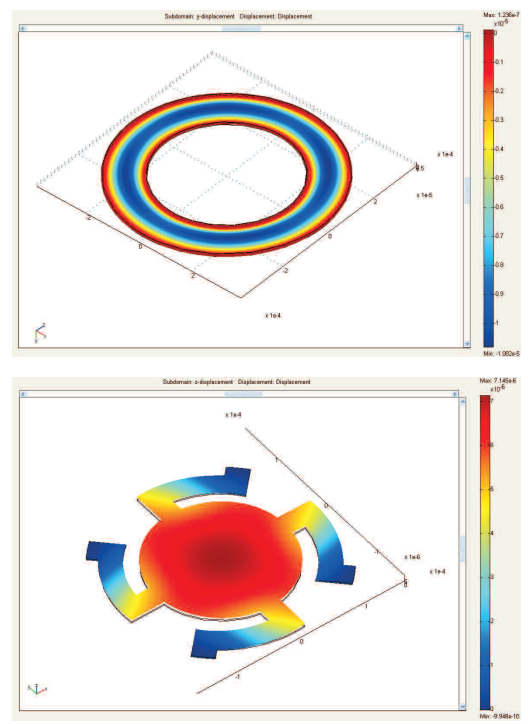

Figure 3. FEMLAB ${ }^{\mathrm{TM}}$ simulations to estimate pre-stressing force of the vacuum-collapsed chamber (top) and resultant threshold pressure applied to valve membrane (bottom) to shunt fluids. By coupling these simulations the dynamic behavior of a complete normally-closed check valve was determined.

\section{FABRICATION}

The fabrication process illustrated in Fig. 4 started with growing a $2 \mu \mathrm{m}$ wet oxide on a standard silicon wafer. The backside of wafer was then bulk-etched by $\mathrm{KOH}$, leaving $25 \mu \mathrm{m}$ membranes for fluidic coupling ports. Afterwards, a multi-layer surface-micromachining technology was utilized to build frontside structures including a series of photoresist and parylene $\mathrm{C}$ layers. The sacrificial photoresist layers were hard-baked at $120{ }^{\circ} \mathrm{C}$ for edge-smoothening and degassing. All parylene $\mathrm{C}$ layers were roughened by oxygen plasma treatment to reduce stiction in the fabricated devices. Using this process, valve seats, check valves, and a integrated microchannel were fabricated. After etching away the backside silicon membrane to open through holes and stripping photoresist with acetone, an additional frontside parylene $\mathrm{C}$ coating was finally applied to accomplish the vacuum-collapsed sealing of the normally-closed valve.
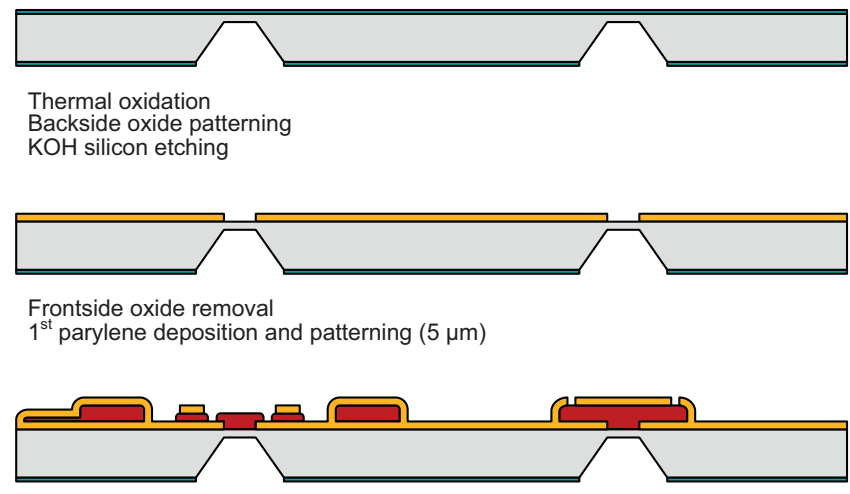

$1^{\text {st }}$ sacrificial photoresist coating and patterning

$2^{\text {nd }}$ parylene deposition and patterning $(2 \mu \mathrm{m})$

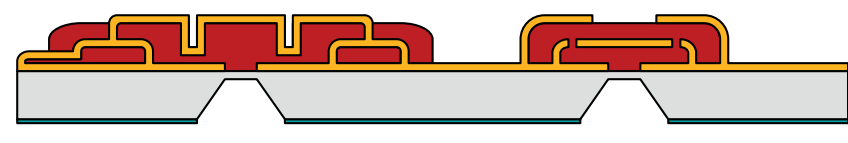

$2^{\text {nd }}$ sacrificial photoresist coating and patterning

$3^{\text {rd }}$ parylene deposition and patterning $(2 \mu \mathrm{m})$

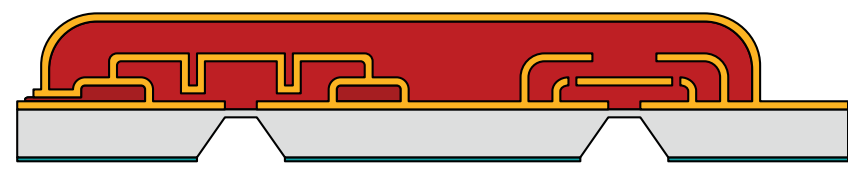

$3^{\text {rd }}$ sacrificial photoresist coating and patterning

$4^{\text {th }}$ parylene deposition and patterning $(5 \mu \mathrm{m})$

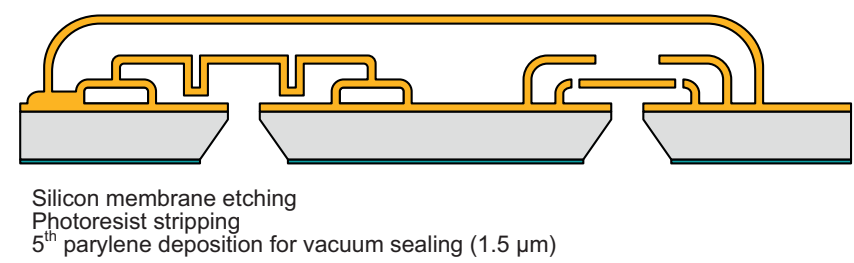

Silicon $\square$ SiO2 $\square$ Parylene $\square$ Photoresist

Figure 4. Fabrication process flow.

During fabrication the normally-closed valve was the most complex component. A three-step partial exposure lithography was applied to a single sacrificial photoresist layer in order to construct a three-level base featuring a vacuum-collapsed chamber, a valve-seat contact, and a etching/sealing channel of a normally- 
closed valve (Fig. 5). A 7- $\mu$ m-high chamber was connected to a 2$\mu \mathrm{m}$-high channel in order to realize final photoresist stripping and $1.5-\mu \mathrm{m}$-thick parylene vacuum sealing through that channel. A 5 $\mu \mathrm{m}$ photoresist spacer between the valve and the valve seat was made to control the pre-stressing force and threshold pressure of the fabricated valve by its height difference to the chamber. Planarization by spin-coating thick photoresist over the three-level structure was then needed to create a flat valve membrane for uniform device performance. Fig. 6 shows the fabricated inchannel parylene dual valves.

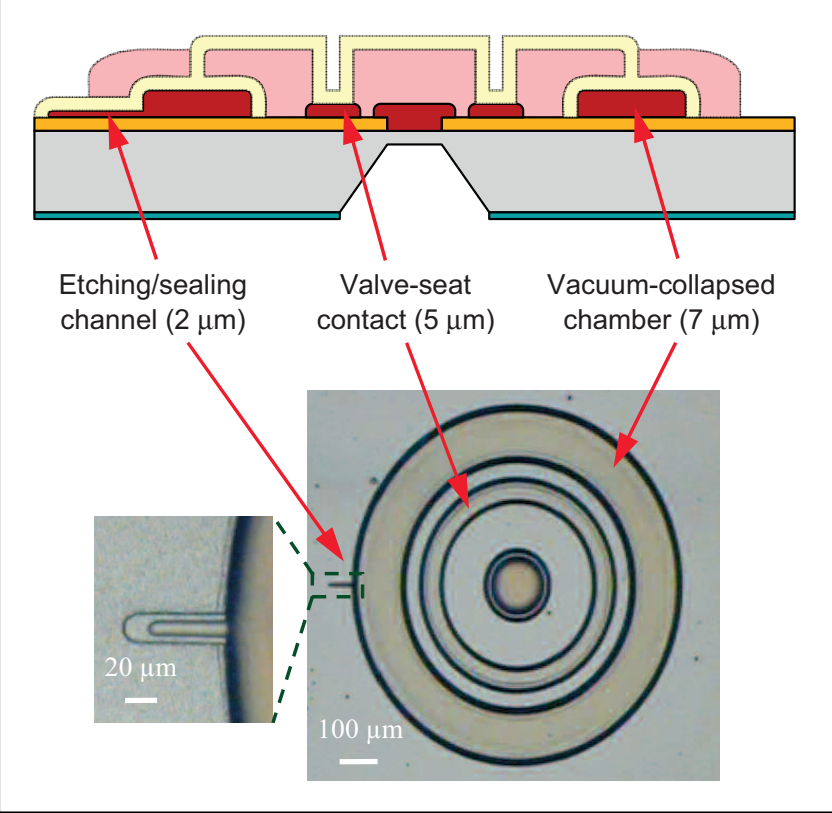

(a)

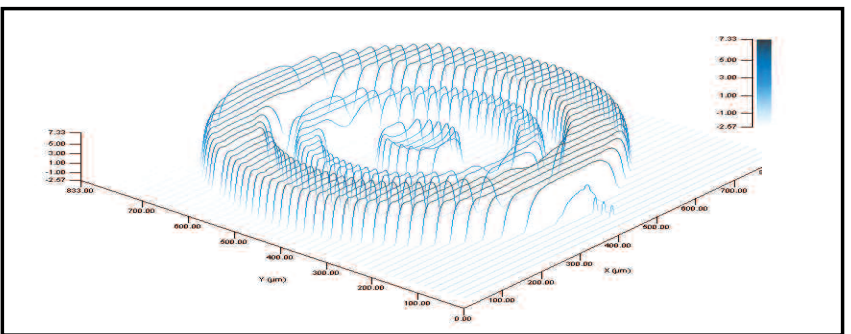

(b)

Figure 5. Three-level sacrificial photoresist layer coating using three-step partial exposure lithography: (a) Cross-section schematic and top-view micrographs of the fabricated structure; (b) 3-D profile scan of (a).
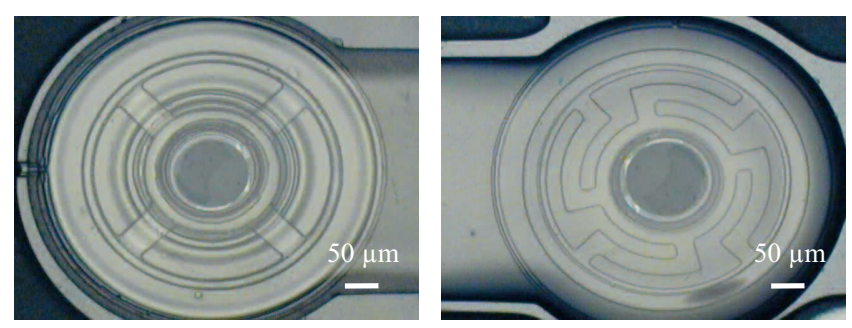

Figure 6. Micrographs of the fabricated in-channel parylene dual valves (top view). (left) Normally-closed micro check valve. (right) Normally-open micro check valve. The diaphragm tethers can be designed as straight arms or twisted arms depending on performance requirements.

\section{TESTING AND DISCUSSION}

Microfabricated in-channel single valves and dual valves were tested using the fluidic setup illustrated in Fig. 7. A customized testing jig was used to provide a macroscale/microscale fluidic interface with diced chips so that microflows could be introduced to the devices through their backside fluidic ports. Compressed air or water served as the fluid source. On the front end of the jig, a pressure regulator and a pressure gauge were connected to control the injection pressure of the fluids. The tuning resolution was up to $0.01 \mathrm{psi}(\sim 0.5 \mathrm{mmHg})$ even without using hydrostatic pressure generated by height difference of liquid column. Flow rate measurement and fluid collection was done on the back end of the jig. Based on the different viscosities of the fluids, flow rate measurement was performed via different methods. Air flow rate was measured with a flow meter due to its large value ( $\mathrm{mL} / \mathrm{min}$ range), while water flow rate was measured by recording the marching velocity of the water column front in the capillary due to its relatively small flow rates (on the order of $\mu \mathrm{L} / \mathrm{min}$ ). Using these techniques, the pressure/flow rate behavior of the devices for the different fluids could be accurately monitored.

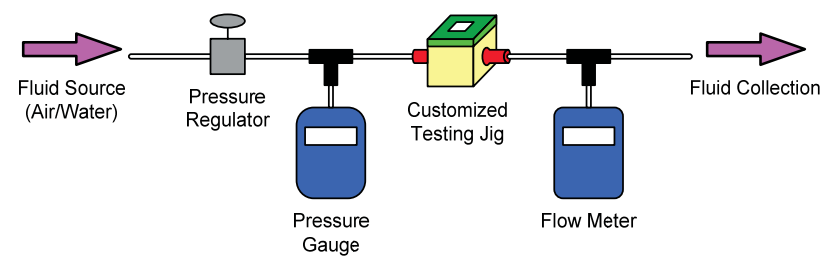

Figure 7. Schematic of fluidic interface setup for device testing.

Pressure/flow rate testing results are shown in Fig. 8 and Fig. 9 using air and water as the working fluids, respectively. The tested valves were of the twisted-arm tether type in order to have a more compliant mechanical response. In single valve operation, the fabricated normally-closed check valve demonstrated a remarkably low cracking pressure around 0.2 psi $(\sim 10 \mathrm{mmHg})$, which was in good agreement with FEMLAB ${ }^{\mathrm{TM}}$ simulations. This was a result of the fluidic pressure being applied on central area of the flexible membrane, thus effectively overcoming the prestressing force of the vacuum-collapsed chamber and shunting the fluids to the channel. Furthermore, there was no backflow leakage to the normally-closed valve. For the normally-open check valve, the cracking pressure was not obviously observed and the forward flow resistance was significantly lower compared to the normallyclosed valve, providing a higher flow rate with the same degree of pressure loading. In terms of reverse leakage, the backward flow rate peaked at more than $2 \mathrm{~mL} / \mathrm{min}$ for air and $2 \mu \mathrm{L} / \mathrm{min}$ for water. This leak rate started to decrease when the pressure loading was higher than $0.6 \mathrm{psi}$ and was greatly suppressed with pressure loading higher than $1 \mathrm{psi}$, which implies that the valve membrane was in firm contact with valve seat. The breakdown pressure was confirmed at more than 25 psi with no obvious leak rate within this range of pressure operation. Microfluidic pressure/flow rate regulation was successfully performed in dual valve operation. Combining the single check valve behaviors, the dual-valved microsystem was able to regulate pressure in a range of $0-1$ psi $(\sim$ $0-50 \mathrm{mmHg}$ ) with measured flow rates of up to $0.7 \mathrm{~mL} / \mathrm{min}$ for air and $1.3 \mu \mathrm{L} / \mathrm{min}$ for water. These small flow rates were due to a large fluidic resistance obtained by the dual check valves with a microchannel in the test configuration. As expected, no reverse leakage was observed with pressure loading greater than 25 psi. 


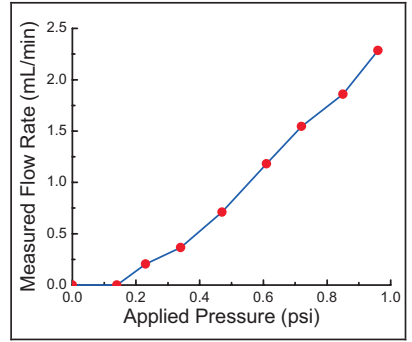

(a)

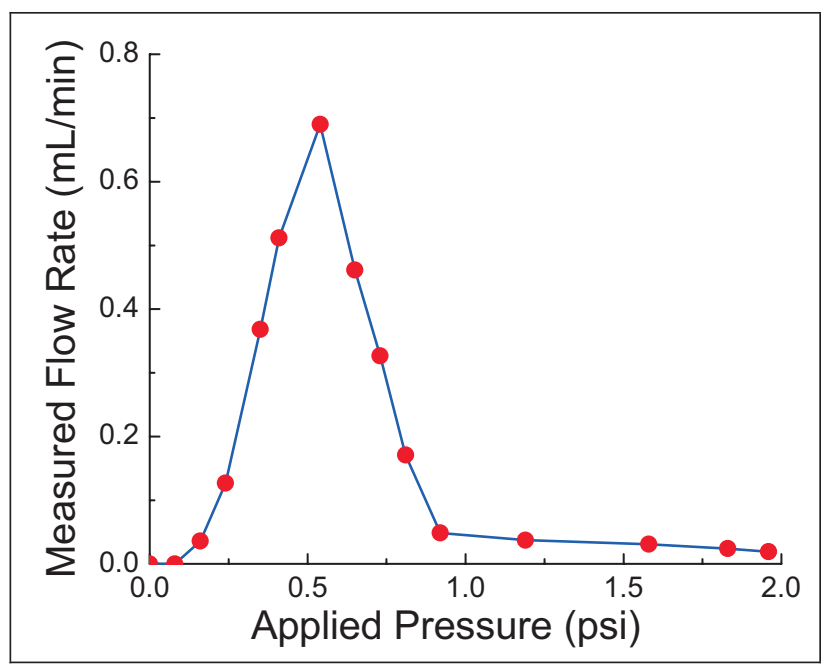

(c)

Figure 8. Device testing with air as the working fluid: (a) Single normally-closed check valve; (b) Single normally-open check valve; (c) Dual valve operation for microflow regulation.

\section{CONCLUSION}

A parylene-based unpowered dual-valved microflow regulation system has been successfully developed. By incorporating a normally-closed and a normally-open check valve in back-to-back configuration inside a microchannel, fluid pressure/flow rate can be systematically controlled. Because of passive operation in check valves, only mechanical elements were involved and no power consumption/transduction was required. A multi-layer surface-micromachining technology was used to construct the integrated dual-valved channels. Fabrication and characterization of single check valves and dual valves have been demonstrated. In single valve testing, both normally-closed and normally-open check valves achieved fluidic behaviors in good agreement with design and simulation. Excellent pressure/flow rate regulation was obtained in dual valve testing. Flow rates of $0.7 \mathrm{~mL} / \mathrm{min}$ for air and $1.3 \mu \mathrm{L} / \mathrm{min}$ for water were obtained within a $0-50 \mathrm{mmHg}$ pressure range. With this unique microflow regulation performance, our biocompatible/implantable dualvalved system can be exploited to conduct life science research and control biological conditions such as regulating elevated IOP of glaucoma patients.

\section{ACKNOWLEDGEMENTS}

This work was supported in part by the Engineering Research Centers Program of the National Science Foundation under NSF Award Number EEC-0310723. The authors especially thank Mr. Trevor Roper for his fabrication assistance.

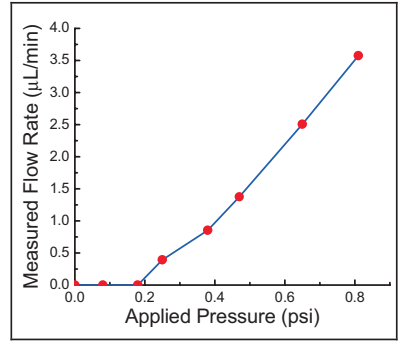

(a)

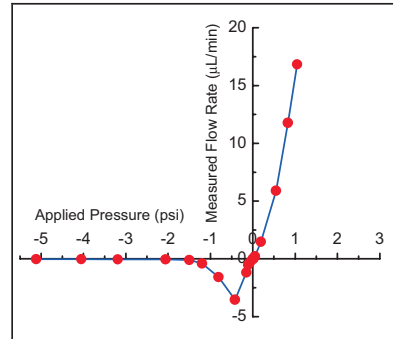

(b)

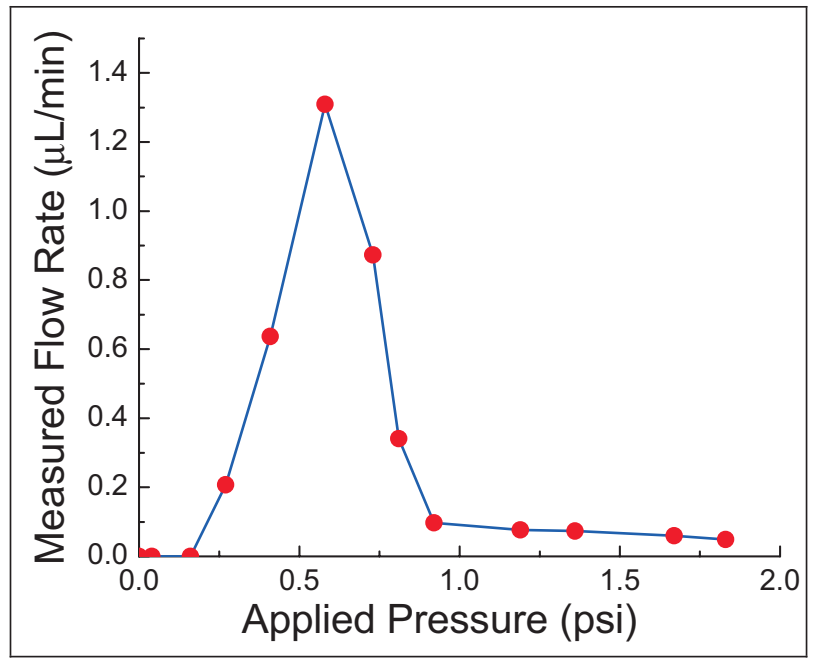

(c)

Figure 9. Device testing with water as the working fluid: (a) Single normally-closed check valve; (b) Single normally-open check valve; (c) Dual valve operation for microflow regulation.

\section{REFERENCES}

[1] A. van den Berg and T. S. J. Lammerink, "Micro Total Analysis Systems: Microfluidic Aspects, Integration Concept and Applications," Topics in Current Chemistry, vol. 194, pp. 21-50, 1997.

[2] S. Shoji, "Fluids for Sensor Systems," Topics in Current Chemistry, vol. 194, pp. 163-188, 1998.

[3] X. Q. Wang, Q. Lin, and Y.-C. Tai, "A Parylene Micro Check Valve," Technical Digest, The 12th IEEE International Conference on MicroElectroMechanical Systems (MEMS 1999), Orlando, FL, USA, Jan. 17-21, 1999, pp. 177-182.

[4] X. Q. Wang and Y.-C. Tai, "A Normally-Closed In-Channel Micro Check Valve," Technical Digest, The 13th IEEE International Conference on MicroElectroMechanical Systems (MEMS 2000), Miyazakli, JAPAN, Jan. 23-27, 2000, pp. 68-71.

[5] J. Xie, X. Yang, X. Q. Wang, and Y.-C. Tai, "Surface Micromachined Leakage Proof Parylene Check Valve," Technical Digest, The 14th IEEE International Conference on MicroElectroMechanical Systems (MEMS 2001), Interlaken, Switzerland, Jan. 21-25, 2001, pp. 539-542. 